\title{
Acknowledgment of Ad Hoc Reviewers
}

The Editor and Associate Editors gratefully acknowledge the following reviewers, who reviewed manuscripts for the 2019 volume.

(⿸丆口

Lise Abrams

Rick Adams

Rashmi Adaval

Trevor Agus

Richard Ahl

Woo-kyoung Ahn

Modupe Akinola

Joe Alba

Genevieve Albouy

Richard J. Allen

Adam Alter

Jamie Amemiya

Michael C. Anderson

Jean-Marie Annon

Ulrich Ansorge

Coren Apicella

Giorgio Arcara

Scott Armstrong

Yuko Arthurs

Richard Artner

Mark H. Ashcraft

Per Aslaksen

Richard Aslin

Kinnari Atit

Anthony Atkinson

Lauren Atlas

Hillel Aviezer

Marios N. Avraamides

Jordan Axt

Ozlem Ayduk

Patric Bach

Shelby Bachman

Gi-Yeul Bae

Melissa Baese-Berk

Bahador Bahrami

Benjamin Baird

Kristen Baker*

Anuenue Baker-Kukona

Benjamin Balas

Christopher T. Ball

Hunter Ball

Daniel Balliet

David Balota

Dan Bang

Bengi Baran

Yoav Bar-Anan

Michael Baratta

Morgan Barense

John Bargh

Michael Barlev

Dave Barner

Andrew Baron

Lisa Barrett

Lawrence Barsalou

Daniel Bartels

Jennifer Bartz

Irina Basieva

Benjamin M. Basile
Ellen Bass

Ilona Bass*

Miriam Bassok

Timothy C. Bates

Laura Batterink

Lucas Battich*

Michael Baumann

Roy Baumeister

Karl-Heinz T. Bauml

Donna M. Bayliss

Paul Bays

C. Philip Beaman

James Bebko

Melissa Beck

Anne-Claude Bedard

James Beebe

Amy Beeston

Eva Belke

Robert Belli

Yitzhak Benbaji

Andrea Bender

Daniel Bennett

Jonah Berger

Dirk Bernhardt-Walther

Dorthe Berntsen

Daniel R. Berry

Bennett I. Bertenthal

Raymond Bertram

Ryan Best

Jamil Palacios Bhanji

Sudeep Bhatia

Rahul Bhui

Lin Bian

Kinsey Bice

Yochanan E. Bigman

Geoff Bird

Wendy Birmingham

James A. Bisby

Robert A. Bjork

David Black

Jessica Black

Caroline Blais

Peter Blake

Iris Blandon-Gitlin

Olaf Blanke

Tashauna Blankenship

Damian Blasi

Katarina Blask

Eliza Bliss-Moreau

Galen Bodenhausen

Elizabeth Bonawitz

Cory Bonn

Walter R. Boot

Andrea Bosco

Mona Roxana Botezatu

Roberto Bottini

Jean-Michel Boucheix

Megan Boudewyn

Mark E. Bouton
Arturo Bouzas

Edward Bowden

Ty W. Boyer

Margaret Bradley

Timothy Brady

William J. Brady

David W. Braithwaite

Mark Brandt

Holly Branigan

Nadia Brashier

Marcel Brass

Todd Braver

Yvonne Brehmer

Eli Brenner

Chris Brewin

Arndt Broder

Christina Brown

Gordon Brown

Nicholas J. L. Brown

Jazmin Brown-Iannuzzi

Maggie Bruck

Susanne Bruckmueller

Tad Brunye

Marc Brysbaert

Tony Buchanan

David Budescu

Eva C. Buechel

Roger Buehler

Marc Jens Buehner

Julie Bugg

Henryk Bukowski

Michael F. Bunting

Juliane Burghardt

A. Mike Burton

Jerome Busemeyer

Lucas Butler

David Buttelmann

Laurel Buxbaum

Katy Cabbage

Luca Campanelli

Linda Camras

Sebastian Cancino

Montecinos*

Katarzyna Cantarero

Jack Cao

Valerio Capraro

Richard Carlson

Alexis Carpenter*

Evan C. Carter*

Eugene M. Caruso

Daniel Casasanto

Elizabeth Casserly

Monica Castelhano

Caroline Catmur

Maria Chait

Kimberly E. Chaney

Hannah H. Chang

Gretchen Chapman
Promothesh Chatterjee

Nikos Chatzisarantis

Robert S. Chavez

Jacqueline M. Chen

Xiaoli Chen

Yi-Chuan Chen

Youguo Chen

Zhansheng Chen

Zoey Chen

Ken Cheng

Yu-Chin Chiu

Eileen Y. Chou

Shamarukh Chowdhury*

Georgia Christodoulou*

Adam Chuderski

Dongil Chung

Jessica Church

Mina Cikara

Andrei Cimpian

Cory Clark

Luke Clark

Berry Claus

Aaron Cochrane*

Jennifer Coffman

Jessica R. Cohen

Leonardo Cohen

Veronica Cole

Alf Coles

John D. Coley

Oliver Collingnon

Benjamin Converse

Paul Conway

Erin Cooley

Gus Cooney

Ingrid M. Cordon

Olivier Corneille

Rense Corten

Albert Costa

Marco Costa

Stéphane Côté

Cathy Cottrell

Jennifer Coull

Sheena Coulson

Marc N. Coutanche

Antoine Coutrot

Elizabeth Crawford

Clayton R. Critcher

Jean-Claude Croizet

Deborah Cronin

Antonietta Curci

Fiery Cushman

Ruud Custers

Hengchen Dai

Ayoub Daliri

Rodica Damian

Claudia Damiano*

Jason Dana

Jennifer E. Dannals 
Shai Davidai

Juliet Davidow

Kathryn Davidson

Jodie Davies-Thompson

Charles Davis*

Ernest Davis

Tyler Davis

Zachary Davis

Clintin Davis-Stober

Telli Davoodi

Jessica De Boer

Felipe De Brigard

Angela de Bruin

Julian De Freitas

Vincent de Gardelle

Linda De Voogd

Greg DeAngelis

Camille Debras

Mathieu Declerck

Egon Dejonckheere

Mauricio Delgado

Chiara Della Libera

Michel Denis

Rachel N. Denison

Nancy Dennis

Jerker Denrell

Nazanin Derakhshan

Jason Deska

Sanford Devoe

Jean-Marc Dewaele

Stephen Dewhurst

Adele Diederich

Edward Diener

Zoltan Dienes

Berkeley Jay Dietvorst

Daniel G. Dillon

Peter H. Ditto

Peter Dixon

Ekaterina Dobryakova

David Dodell-Feder

Myrthel Dogge

Clara Dominke*

Sara Loughran Dommer

Kristin Donnelly

Brian Donovan

Jennifer Donovan

Bruce Dore

Fabrizio Doricchi

Michael R. Dougherty

Igor Douven

John Dovidio

W. Jay Dowling

Dejan Draschkow

Noemi Dreksler

Sarah DuBrow

Brad Duchaine

Eilish Duke

John Duncan

Katherine Dawn Duncan

Yarrow Dunham

Timothy Lee Dunn

David A. Dunning

Joseph Dunsmoor

Cydney H. Dupree
Lily Durwood*

Carol Dweck

Allison Earl

Ullrich Ecker

Mark Edwards

Howard Egeth

Boris Egloff

Tobias Egner

Richard Eibach

Andrew Elliot

Emily M. Elliott

William Emeny*

Karen Emmorey

Stephen Emrich

Laura E. Engelhardt

Haakon Engen

Christoph Englert

Birte Englich

James T. Enns

Christopher D. Erb

Ido Erev

Michael Esterman

Zachary Estes

Nuria Esteve-Gibert

Ulrich Ettinger

Ioannis Evangelidis

Jonathan Evans

Anthony M. Evans

Nathan John Evans

Jim A. C. Everett

Louise Ewing

Joel Fagot

Yana Fandakova

Xiaoping Fang*

George D. Farmer

Lisa Fazio

Russell H. Fazio

Aidan Feeney

Lisa Feigenson

Daniel Feiler

Qianli Feng

Heather Ferguson

Victor Ferreira

Francesca Ferri

Evelyn C. Ferstl

Ian Fiebelkorn

Klaus Fiedler

Katrina Marie Fincher

Chaz Firestone

Ilan Fischer

Jason Fischer

József Fiser

Ayelet Fishbach

Cynthia Fisher

Ronald Fisher

Ahren Fitzroy*

William Fleeson

Roland Fleming

Colin Flynn

Charles Folk

Brett Q. Ford

Jaclyn Ford
Donelson Forsyth

Omid Fotuhi

Abdellah Fourtassi*

Kyriaki Fousiani

Candice Frances

John Franchak

Wendy S. Francis

David Joseph Frank

Scott H. Fraundorf

Megan Freeth

Renato Frey

Ori Friedman

Jeremy Frimer

Christian Frings

Karl Friston

Chris D. Frith

Romy Fromer

Keisuke Fukuda

Celia Gaertig

Brendan Gaesser

Sarah Gaither

Fabio Galeotti

Mirta Galesic

Giovanni Galfano

David Gallo

Adriana Galvan

Matthias Gamer*

Giorgio Ganis

Zaifeng Gao

Leire Gartzia

Susan Gathercole

Brandy Gatlin-Nash

Danielle Gaucher

Isabel Gauthier

Bertram Gawronski

Andra Geana

Charles Geier

Susan Gelman

Joy Geng

Silvia Gennari

J. P. Gerber

Christian Gerlach

Richard Gerrig

Sarah Gerson

Tobias Gerstenberg

Miranda Giacomin

Carina Giesen

Daniel Gilbert

Beatriz Gil-Gomez de Liano

Thomas Gilovich

Fabio Giommi

Pascale Gisquet-Verrier

Nathalia Gjersoe

Elizabeth Glisky

Andreas Gloeckner

Sebastian Gluth

Lisa Goffman

Amit Goldenberg

Morris Goldsmith

Thalia Goldstein

Tamar Gollan

Rebecca Gomez

Corentin Gonthier
Stephanie Goodhew

Geoffrey Goodwin

Mira Goral

Amie Gordon

Peter Gordon

Jacqueline Gottlieb

Martijn Goudbeek

Annabelle Goujon

Arthur Graesser

Gabriele Gratton

Oliver Gray

David Green

Shawn C. Green

Robert Greene

Anthony G. Greenwald

Lindy Greer

Paul Gribble

Saskia Giebl*

Tom Griffiths

Anastasiia Grigoreva*

Quentin Frederik Gronau

Maddie Groom

Jörg Gross

Igor Grossmann

Matthias Gruber

Tricia Guerrero*

Selin Gulgoz

Jason Gullifer

Prahlad Gupta

Suzanne Gurland

Juan Guevara Pinto*

Arvid Guterstam

Julia M. Haaf

Jan Haaker

Leor M. Hackel

Steven Hackley

Nobuhiro Hagura

Adam Hahn

Jonathan Haidt

May Ling D. Halim

Judy Hall

Zach Hambrick

Kiley Hamlin

Simon Handley

Paul H. P. Hanel

Deborah Hannula

Simon Hanslmayr

David J. Hardisty

Oliver Hardt

Adam Harris

Catherine Harris

Paul L. Harris

Cate Hartley

Tom Hartley

Michael Haselhuhn

Nick Haslam

Ran Hassin

Reid Hastie

Markus Hausmann

Robert Hawkins

Brett K. Hayes

Grace Hayes*

Taylor R. Hayes 
Lisheng $\mathrm{He}$

Sheng $\mathrm{He}$

Andrew Heathcote

Joseph Heffner

Peter Hegarty

Eric Hehman

Steven Heine

Larisa Heiphetz

Agneta Herlitz

Rachel Hershenberg

Paula T. Hertel

Ralph Hertwig

Alexis Hervais-Adelman

Neil Hester

Jason Hicks

Sarah E. Hill

Thomas Hills

Mark Himelstein*

Graham Hitch

Arnold Ho

Cristy Ho

Sara Hodges

Katie Hoemann*

Ulrich Hoffrage

Markus J. Hofmann

Robin M. Hogarth

Tim Hollins

Kevin James Holmes

Nicholas P. Holmes

Bruce Homer

Bernhard Hommel

Sang Hong

Zachary Horne

Aidan Horner

William S. Horton

Joseph Houpt

Philippa L. Howard

Piers Howe

Daniel Hruschka

Christopher Hsee

Janet Hui-wen Hsiao

Ming Hsu

Joel Huber

Kylie Huckleberry

Nathan Hudson

Robert Hughes

Alycia Hund

Cendri Hutcherson

J. Benjamin Hutchinson

Hans IJzerman

Hee Yeon Im

Yoel Inbar

Helene Intraub

Muireann Irish

Julia Irwin

Ivan Ivanchei

Rich Ivry

Joshua Jackson

W. Jake Jacobs

Nir Jacoby

Tomasz Jankowski

Steve M. J. Janssen
Nicole Jardine

Agnieszka Jaroslawska

Andrew F. Jarosz

Chris Jarrold

Shabnam Javdani

Adrianna C. Jenkins

Li-Jun Ji

Tonglin Jiang*

Leslie John

Camille Johnson

Eric Johnson

Matt Jones

Michael Jones

Jillian Jordan

Holly Joseph

Eric Juarez*

Mark Jung-Beeman

Georgiana Juravle

Alexandros Kafkas

Hemant Kakkar

Zsuzsa Kaldy

Tobias Kalenscher

Sunjeev Kamboj

Yoshihisa Kashima

Barbara Kaup

Jun Kawahara

John Raymond Keefe

Mike Keesman

Frank Keil

David Kellen

Colleen Kelley

Chris Kello

Simon Kelly

Gideon Keren

Nicolas Kervyn

Dirk Kerzel

Thomas Kessler

Boaz Keysar

Adam Kiefer

Markus Kiefer

Andrea Kiesel

Rogier Kievit

Melanie Killen

B. Kyu Kim

Emily Kim*

Kevin King

K. Christoph Klauer

Sabina Kleitman

Chris Klink

Ewelina Knapska

Joshua Knobe

Guenther K. Knoblich

Barbara Knowlton

Alex Koch

Melissa Koenig

Stefan Kohler

Agnieszka Konopka

Ana Konova

Wouter Kool

Catalina Kopetz

Bogdan Kostic

Yubo Kou

Maryam Kouchaki
John Kounios

Margaret Kovera

Maria Kozhevnikov

Veronika Kraft*

E. J. Krahmer

Ian Krajbich

Hannah Kramer*

Ralf Krampe

Max Krasnow

Michael Kraus

Robert Kreuzbauer

Anand Krishna

Amy Krosch

Brice A. Kuhl

Christopher A. Kurby

Benedek Kurdi

Didem Kurt

Kenneth Kurtz

Florian Kutzner

Lia Kvavilashvili

Patrick C. Kyllonen

Jordan LaBouff

Kristin Hansen Lagattuta

David A. Lagnado

Hayley Lagroix

Calvin K. Lai

Brenden Lake

Azara Lalla*

Amrita Lamba

Anthony J. Lambert

Joris Lammers

James Lampinen

Mark Landau

Dominic Landgraf

Justin F. Landy

Richard Lane

Florian Lange

Jens Lange

Stephen R. H. Langton

Richard Larrick

Bethany Lassetter*

Hakwan Lau

Sean Laurent

Kristin Laurin

Nadine Lavan

Michal Lavidor

Aureliu Lavric

Bonnie Le

Gael Le Mens

Mike Le Pelley

Brooke Lea

Marius Leckelt

Alison Ledgerwood

Kang Lee

Sang Ah Lee

Jeffrey Lees*

Ryan Lee-James

Jo-Anne LeFevre

Cristine Legare

Michelle Leichtman

Ed Lemay

Karolina Lempert

Heather C. Lench
Dorothea Lerman

Jennifer Susan Lerner

Benjamin Levy

Ashley Glen Lewis

Jarrod Lewis-Peacock

Nira Liberman

Zoe Liberman

Klaus Libertus

Melissa Libertus

Margarita Lieb

Heinrich René Liesefeld

Scott Lilienfeld

Aedan $\mathrm{Li}^{*}$

Hause Lin

Tian Lin

Sam Ling

Angelika Lingnau

Daniel R. Little

Jonathan Livengood

Evan Livesey

Kimberly Livingstone

Alejandro Lleras

Emily Paige Lloyd

Vanessa LoBue

Patricia Lockwood

George Loewenstein

Shayne Loft

Gordon D. Logan

Lynn Lohnas

Tania Lombrozo

Matthew Longo

Antonella Lopez ${ }^{*}$

Lester Loschky

Bradley Love

Chunming Lu

Richard E. Lucas

Steven Luck

Elliot A. Ludvig

Roy Luria

Andrew Luttrell

Ian M. Lyons

John Mace

Edouard Machery

Laura Machia

C. Neil Macrae

Jens Madsen

Sam Maglio

Cyrille Magne

James Magnuson

Eleanor Anne Maguire

Steve Majerus

Tal Makovski

Mora Maldonado

Simone Malejka

Selin A. Malkoc

Remington Mallett*

Kenneth Malmberg

Tara Mandalaywala

David R. Mandel

Kristan Marchak

Alex Marchant

Isabelle Mareschal

Julian Marewski 


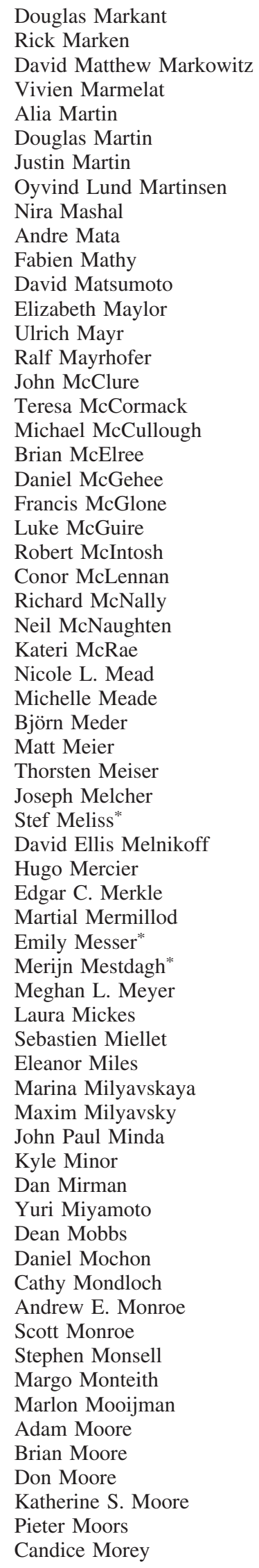

Alexandra Morrison

Hannah Morrow*

Kinga Morsanyi

Riikka Mottonen

Michael C. Mozer

Liad Mudrik

Tim Mullett

Neil W. Mulligan

Kelly Lynn Mulvey

Kou Murayama

Charlotte Murphy*

Peter R. Murphy

Vishnu Murty

Thomas Mussweiler

Kristian Myrseth

\section{Marcos Nadal \\ Andrew Neal \\ Ian Neath \\ Elizabeth Necka* \\ Amy Needham \\ Rebecca Neel \\ W. Trammell Neill \\ Julie Neiworth \\ Adrian Nestor \\ Heiko Neumann \\ Nora Newcombe \\ Ben Newell \\ Florrie Ng \\ Tiffani $\mathrm{Ng}^{*}$ \\ Rosalind Nguyen* \\ Shaun Nichols \\ Jessica Nicosia* \\ Gavin Nobes \\ Jean-Paul Noel \\ Simon Nørby \\ Ann Nordmeyer \\ Nathan Novemsky \\ Alexander Noyes* \\ Eilidh Noyes}

Mike Oaksford

Natalie A. Obrecht Lindemann

Ed O'Brien

John P. O'Doherty

Aileen Oeberst

Christopher Olivola

Maria Olkkonen

Kristina Olson

Michael Olson

Dilek Onkal

John Opfer

Edward Orehek

Gerardo Ortega

Javier Ortiz-Tudela*

Ariana Orvell

Magda Osman

Frederick Oswald

Ross Otto

Daphna Oyserman

Kenneth Paap

Keith Paarporn

Amy Pace
Thorsten Pachur

Neeru Paharia

Asa Palley

Matthew Palmer

Stefano Palminteri

Josefa Pandeirada

Mario Pandelaere

Megan H. Papesh

Esther Papies

Hyeongdong Park*

Seongmin Park

Sohee Park

Jeffrey R. Parker

Colleen Parks

Richard Parncutt

Harold Pashler

Sona Patel

Kevin Paterson

Keith Payne

Eric J. Pedersen

Caterina Pedersini*

Johanna Peetz

Didem Pehlivanoglu

Sergio Pellis

Mateo Penaherrera*

Gordon Pennycook

Francesca Peressotti

Jasmin Perez*

Mario Borja Perez*

Koraly Perez-Edgar

Ricardo Perez-Truglia

Hannah Perfecto

Charles Perfetti

Susan Perlman

Olivia Petit

Richard E. Petty

Penny M. Pexman

Andrea Philipp

Jonathan Phillips

Curtis Edward Phills

Alan Pickering

Paul Piff

David Pillemer

Andre Pittig

Jane Plailly

Jason Plaks

Timothy J. Pleskac

Yehuda Pollak

Evan Polman

Daniel Poole

Diane Poulin-Dubois

Lindsey Powell

Jay Pratt

Michael S. Pratte

Drazen Prelec

Daniel Pressnitzer

Jon Prince

John Protzko

Wojtek Przepiorka

Mita Puri

Timothy Pychyl

Kimberly Quinn

Jordi Quoidbach
Adam Qureshi

Gabriel Radvansky

Candace Raio

Suparna Rajaram

Gerardo Ramirez

Gary Raney

Antonio Rangel

David N. Rapp

Roger Ratcliff

Jane E. Raymond

Jenny Read

Rolf Reber

Thomas Redick

Diego Reinero

Roger Remington

Peter Rendell

Frank Renkewitz

Kathleen Pirog Revill

Alodie Rey-Mermet

V. F. Reyna

Kate Reynolds

Bert Reynvoet

Stephen Rhodes

Marjorie Rhodes

Francois Ric

Tobias Richter

Megan Ringel*

Lance Rips

Jane Risen

Evan Risko

Stuart Ritchie

Rachel Robbins

Steven O. Roberts

Kim P. Roberts

Oliver Robinson

Jennifer Rodd

Angelo Romano

Laura Rosseel

Shannon Ross-Sheehy

Kathrin Rothermich

Klaus Rothermund

Constantin Rothkopf

Joshua Rottman

Jeffrey N. Rouder

Jay Rueckl

Markus Ruetgen

Ian Ruginski*

Jan Rummel

James A. Russell

Jay Edward Russo

Adam Rutland

Rachel Ryskin

Mark Sabbagh

Aaron M. Sackett

Brad Sagarin

Sunita Sah

Lili Sahakyan

Giovanni Sala

Jessica Salerno

Gregory Samanez-Larkin

Elisa Santandrea

Marc Sato 
Ajay Satpute

Blair Saunders

Kristin (Grunewald) Saunders*

Krishna Savani

Daniel Schacter

Stacey Schaefer

Chelsea Schein

Dan Schley

Meg Schlichting

Constance Schmidt

Kathleen Schmidt

Daniel Schmidtke

Bruce Schneider

Dana Schneider

Darryl Schneider

Katharina Schnitzspahn

Felix Schönbrodt

Gregor Schöner

Jonathan Schooler

Lael Schooler

Juliana Schroeder

Sara E. Schroer*

Thomas Schubert

Michael Schulte-Mecklenbeck

Christin Schulze

Mark Schurgin

Amos Schurr

Michael Schutz

Janet Schwartz

Maurice Schweitzer

Shane Schweitzer

Carol Seger

Colleen M. Seifert

Paul Seli

Gun Semin

Julio Sevilla

Ovul Sezer

Alexander Shackman

Victoria Shaffer

Priti Shah

Shaul Shalvi

David R. Shanks

Azim Sharif

Alex Shaw

Paschal Sheeran

Stephen Sheinkopf

Signy Sheldon

Amitai Shenhav

Jeff Sherman

Maxine Sherman

Mark Sheskin

Grant S. Shields

Richard Shiffrin

Yee Lee Shing

Thomas Shipley

David Shore

Garriy Shteynberg

Andrew Shtulman

Suzanne Shu

Kristin Shutts

David Sidhu*

Noam Siegelman

Robert Siegler

Carol K. Sigelman
Dan Simon

Uri Simonsohn

Murray Singer

Linda J. Skitka

John J. Skowronski

Timothy Slattery

Michael L. Slepian

Robert Sleve

Vladimir Sloutsky

Annique Smeding

Bridget Smeekens

Daniel Smilek

Luke Smillie

Colin Smith

Steven M. Smith

Mojtaba Soltanlou

Mitch Sommers

Antonella Sorace

Alexander Soutschek

Alessandra S. Souza

Sophie Sowden

Thomas M. Spalek

Michael Spezio

Sanjay Srivastava

Christoph Stahl

Ariel Starr

Piers Steel

Catherine Steele

Mary Steffel

Timo Stein

Jennifer Stellar

Elena Stepanova

Simon Stephan

Philip Sterzer

Neil Stewart

Benjamin Storm

Bryan Strange

Brent Strickland

Kristof Strijkers

Samantha Strong

Yoann Stussi

Sumarga Suanda

Kristina Suchotzki

Daniel Sullivan

Tina Sundelin

Tirta Susilo

Clare A. M. Sutherland

Keisuke Suzuki

Steven Sweldens

Koji Takahashi*

Melanie Takarangi

Simone Tang

Ben Tappin

Vince Taschereau-Dumouchel

Arber Tasimi

J. S. H. Taylor

Kristina Tchalova*

Yuri Terasawa

Lore Thaler

Dustin Thoman

Valerie Thompson

Mark Thornton

Laura Tian
Jeggan Tiego

Scott Tindale

Jason Tipples

Mehrgol Tiv

Christopher To

Stephen Tobin

Sascha Topolinski

Rima Toure-Tillery

James Townsend

Sarah Townsend

Stefan Trautmann

Sophie Trawalter

Jennifer Trueblood

Konstantinos Tsetsos

Brandon Turner

Brad Turnwald

Joshua Tybur

Lucina Uddin

Tomer Ullman

Rolf Ulrich

Oyku Uner*

Christian Unkelbach

Nash Unsworth

Oleg Urminsky

Marius Usher

Igor Utochkin

David Uttal

Miguel Vadillo

Robin R. Vallacher

Jeroen Van Baar

Jeroen van Boxtel

Ineke van der Ham

Sander van der Linden

Stefan Van der Stigchel

Pieter Van Dessel

Florian Van Leeuwen

Caspar van Lissa

Carien van Reekum

Henk van Steenbergen

Wijnand Adriaan Pieter Van Tilburg

Jeffrey Vancouver

Joachim Vandekerckhove

Christina Vanden Bosch der Nederlanden

Signe Vangkilde

Ivan Vargas

Paul Verhaeghen

Tessa Verhoef

Gabriella Vigliocco

Sagana Vijayarajah*

Navin Viswanathan

Edward K. Vogel

Erin Vogel

Tobias Vogel

Julia Vogt

Claudia Christina von Bastian

Andrew Vonasch

Jennifer Vonk

Ed Vul
Merle Wachendörfer*

Eric-Jan Wagenmakers

Cheryl Wakslak

Lukasz Walasek

Drew Walker

Eric A. Walle

Felix Wang

Ke Wang*

Geoff Ward

Margaret Wardle

Lara Warmelink

Shoko Watanabe

Amanda Helen Waterman

Joseph Watts

Sandra Waxman

John Wearden

Michael Weinstock

Gary Wells

Dorit Wenke

Dirk Wentura

Andrew Westbrook

Chris F. Westbury

Mark Whisman

Peter A. White

Sarah White

Douglas Whiteside

David Whitney

Sherri Widen

Lutz Wiegrebe

Reinout Wiers

Michał Wierzchoń

Holger Wiese

Ronnie Wilbur

Jennifer Wiley

Oliver Wilhelm

Craig Williams

John Wilson

Bo Winegard

Daniel Wisneski

Maria Witek

Marco Wittmann

Thomas Wolbers

Benjamin Wolfe

Jeremy Wolfe

Phillip Wolff

Aaron Wong

Adrienne Wood

Wendy Wood

Geoffrey F. Woodman

Evy Woumans

Sherry Jueyu Wu

Brad Wyble

Jordana Wynn

Hong Xu

Yaoda Xu

Ofir Yakobi*

Allison Yamanashi Leib

Jeremy Yamashiro

Suhui Yap*

Arash Yazdanbakhsh

David Yeager* 
Eldad Yechiam

Eiling Yee

Kathryn Yee*

Nick Yeung

Henry Yin

Daniel Yon

Liane Young
Galit Yovel

Yossi Yovel

Chen Yu

Carole Yue

Jamil Zaki

Safa Zaki
Anthony P. Zanesco

Rene Zeelenberg

Dasa Zeithamova

John Zelenski

Jay Zenkic*

Thomas Zentall

Han Zhang*
Weiwei Zhang

Jiaying Zhao

Hubert Zimmer

Vladimir Zotov

Sascha Zuber

Jennifer Zuk

* Denotes a reviewer who co-reviewed under the supervision of a primary reviewer. 